\title{
The flux-weighted gravity-luminosity relation of Galactic classical Cepheids ${ }^{\star}$
}

\author{
M. A. T. Groenewegen
}

Koninklijke Sterrenwacht van België, Ringlaan 3, 1180 Brussels, Belgium

e-mail: martin.groenewegen@oma.be

Received 29 April 2020 / Accepted 22 June 2020

\begin{abstract}
The flux-weighted gravity-luminosity relation (FWGLR) is investigated for a sample of 477 classical Cepheids (CCs), including stars that have been classified in the literature as such but are probably not. The luminosities are taken from the literature, based on the fitting of the spectral energy distributions (SEDs) assuming a certain distance and reddening. The flux-weighted gravity (FWG) is taken from gravity and effective temperature determinations in the literature based on high-resolution spectroscopy. There is a very good agreement between the theoretically predicted and observed FWG versus pulsation period relation that could serve in estimating the FWG (and $\log g$ ) in spectroscopic studies with a precision of 0.1 dex. As was known in the literature, the theoretically predicted FWGLR relation for CCs is very tight and is not very sensitive to metallicity (at least for LMC and solar values), rotation rate, and crossing of the instability strip. The observed relation has a slightly different slope and shows more scatter ( 0.54 dex). This is due both to uncertainties in the distances and to the pulsation phase averaged FWG values. Data from future Gaia data releases should reduce these errors, and then the FWGLR could serve as a powerful tool in Cepheid studies.
\end{abstract}

Key words. stars: distances - stars: fundamental parameters - stars: variables: Cepheids

\section{Introduction}

Classical Cepheids (CCs) are considered an important standard candle because they are bright, and thus they comprise a link between the distance scale in the nearby universe and that further out via those galaxies that contain both Cepheids and SNIa (see Riess et al. 2019 for a determination of the Hubble constant to $1.9 \%$ precision, taking into account the new $1.1 \%$ precise distance to the Large Magellanic Cloud from Pietrzyński et al. 2019).

This is the third paper in a series on Galactic CCs based on the Gaia second data release (GDR2, Gaia Collaboration 2018). Groenewegen (2018) (hereafter G18) started from an initial sample of 452 Galactic CCs with accurate $[\mathrm{Fe} / \mathrm{H}]$ abundances from spectroscopic analysis. Based on parallax data from Gaia DR2, supplemented with accurate non-Gaia parallax data when available, a final sample of about $200 \mathrm{FU}$ mode Cepheids with good astrometric solutions was retained to derive periodluminosity $(P L)$ and period-luminosity-metallicity $(P L Z)$ relations. The influence of a parallax zeropoint offset on the derived $P L(Z)$ relation is large and means that the current GDR2 results do not allow to improve on the existing calibration of the relation or on the distance to the LMC (as also concluded by Riess et al. 2018). The zeropoint, the slope of the period dependence, and the metallicity dependence of the $P L(Z)$ relations are correlated with any assumed parallax zeropoint offset.

In Groenewegen (2020) (hereafter G20) the sample was expanded to 477 stars. Using photometry over the widest

* Full Table A.3 is only available at the CDS via anonymous ftp to cdsarc.u-strasbg. fr (130.79.128.5) or via http://cdsarc. u-strasbg.fr/viz-bin/cat/J/A+A/640/A113 available range in wavelength (and at mean light when available) the spectral energy distributions (SEDs) were constructed and fitted with model atmospheres (and a dust component when required). For an adopted distance and reddening these fits resulted in a best-fitting bolometric luminosity $(L)$ and the photometrically derived effective temperature $\left(T_{\text {eff }}\right)$. This allowed for the derivation of period-radius $(P R)$ and $P L$ relations, the construction of the Hertzsprung-Russell diagram (HRD), and a comparison to theoretical instability strips (ISs). The position of most stars in the HRD was consistent with theoretical predictions. Outliers were often associated with sources where the spectroscopically and photometrically determined effective temperatures differed, or with sources with large and uncertain reddenings.

In this paper the relation between bolometric absolute magnitude and the flux-weighted gravity (FWG), $g_{\mathrm{F}} \sim g / T_{\mathrm{eff}}^{4}$, is investigated: the so-called flux-weighted gravity-luminosity relation (FWGLR). The tight correlation between $g_{\mathrm{F}}$ and luminosity was first demonstrated by Kudritzki et al. (2003, 2008) for blue supergiants, and was then used for extragalactic distance determinations in Kudritzki et al. (2016). Anderson et al. (2016) demonstrated that theoretical pulsation models for CCs also followed a tight FWGLR, in fact tighter than the $P L$ relation, and that there was a good correspondence between observed $g_{\mathrm{F}}$ and period for a sample of CCs. The latest calibration of the FWGLR is presented in Kudritzki et al. (2020) based on 445 stars ranging from $M_{\mathrm{bol}}=+9.0$ to -8.0 .

The paper is structured as follows. In Sect. 2 the theoretical models of Anderson et al. (2016) are compared to the latest calibration in Kudritzki et al. (2020). In Sect. 3 the sample of 477 (candidate) CCs is introduced and the $g_{\mathrm{F}}$ are derived, and the correlation with period and luminosity are presented. A brief discussion and summary concludes the paper. 


\section{Theoretical FWGLR for CCs}

The FWG is defined as $\log g_{\mathrm{F}}=\log g-4 \cdot \log \left(T_{\text {eff }} / 10^{4}\right)$ (Kudritzki et al. 2003). Kudritzki et al. (2020) present the latest calibration of the FWG against absolute bolometric magnitude as

$M_{\mathrm{bol}}=(3.19 \pm 0.01)\left(\log g_{\mathrm{F}}-g_{\mathrm{F}}^{\odot}\right)+(4.74 \pm 0.01)$

for $\log g_{\mathrm{F}} \geq \log g_{\mathrm{F}}^{\mathrm{b}}$ and

$M_{\mathrm{bol}}=(3.76 \pm 0.11)\left(\log g_{\mathrm{F}}-g_{\mathrm{F}}^{\mathrm{b}}\right)+(-2.98 \pm 0.09)$

for $\log g_{\mathrm{F}}<\log g_{\mathrm{F}}^{\mathrm{b}}$, with a scatter of 0.17 and $0.29 \mathrm{mag}$, respectively. The break in the relation is set at $g_{\mathrm{F}}^{\mathrm{b}}=3.0$, while the FWG of the Sun is $g_{\mathrm{F}}^{\odot}=5.39$.

Anderson et al. (2016) presented a large set of pulsation models for CCs based on stellar evolutionary models for a range of initial masses $\left(1.7-15 M_{\odot}\right)$, initial rotation rates $\left(\omega_{\text {ini }}=0.0\right.$, $0.5,0.9$ in terms of the critical rotation rates), metallicities $(Z=$ $0.002,0.006,0.014)$, and for fundamental mode (FU) and first overtone (FO) CCs. Stellar parameters $\left(L, T_{\text {eff }}\right)$, and pulsation periods are given at the entry and exit of the IS for various crossings. They used these models to show the tight FWGLR for CCs for the first time (Fig. 16 in Anderson et al. 2016).

The top panel in Fig. 1 shows the theoretical FWGLR based on these models for FU pulsators with periods $>0.6 \mathrm{~d}$, FO pulsators with periods $>0.4 \mathrm{~d}, Z=0.006$ and 0.014 , and all rotation rates and crossings of the IS as the coloured lines and symbols. Also shown are Eqs. (1) and (2). For the lower gravities the models deviate from Eq. (2), and appear to be closer to an extension of Eq. (1). A linear fit to these models gives the relation

$M_{\mathrm{bol}}=(3.35 \pm 0.02)\left(\log g_{\mathrm{F}}-g_{\mathrm{F}}^{\mathrm{b}}\right)+(-2.975 \pm 0.012)$

with an rms of $0.16 \mathrm{mag}$, shown as the green line in the figure. Additional fits are given in Appendix A.

The bottom panel shows the relation between FWG and period for the same selection of models (cf. Fig. 17 in Anderson et al. 2016, 2020). Periods of FO models are fundamentalised using the relation $P_{0}=P_{1} /\left(0.716-0.027 \log P_{1}\right)$ following Feast \& Catchpole (1997). The best fit is

$\log g_{\mathrm{F}}=(-0.834 \pm 0.011) \log P_{0}+(3.402 \pm 0.011)$

with an rms of 0.09 dex. Eliminating the second crossing models would result in a fit with a smaller scatter, but as this information is not known a priori the relation as presented is more generally applicable when an estimate of $\log g_{\mathrm{F}}$ is desired. Figures and relations for FU and FO models separately are presented in the appendix.

\section{Sample and observed FWGs}

The sample studied here is the collection of 477 stars considered in G20. It is based on the original sample of 452 stars compiled in G18, extended by 25 additional stars for which accurate iron abundances have since become available, including five CCs in the inner disk of our Galaxy (Inno et al. 2019).

G20 constructed the SEDs for these stars, considering photometry from the ultraviolet to the far-infrared, and as much as possible at mean light. Distances and reddening were collected from the literature. Distances from GDR2 data was available for 232 sources, and from other parallax data for 26 stars.
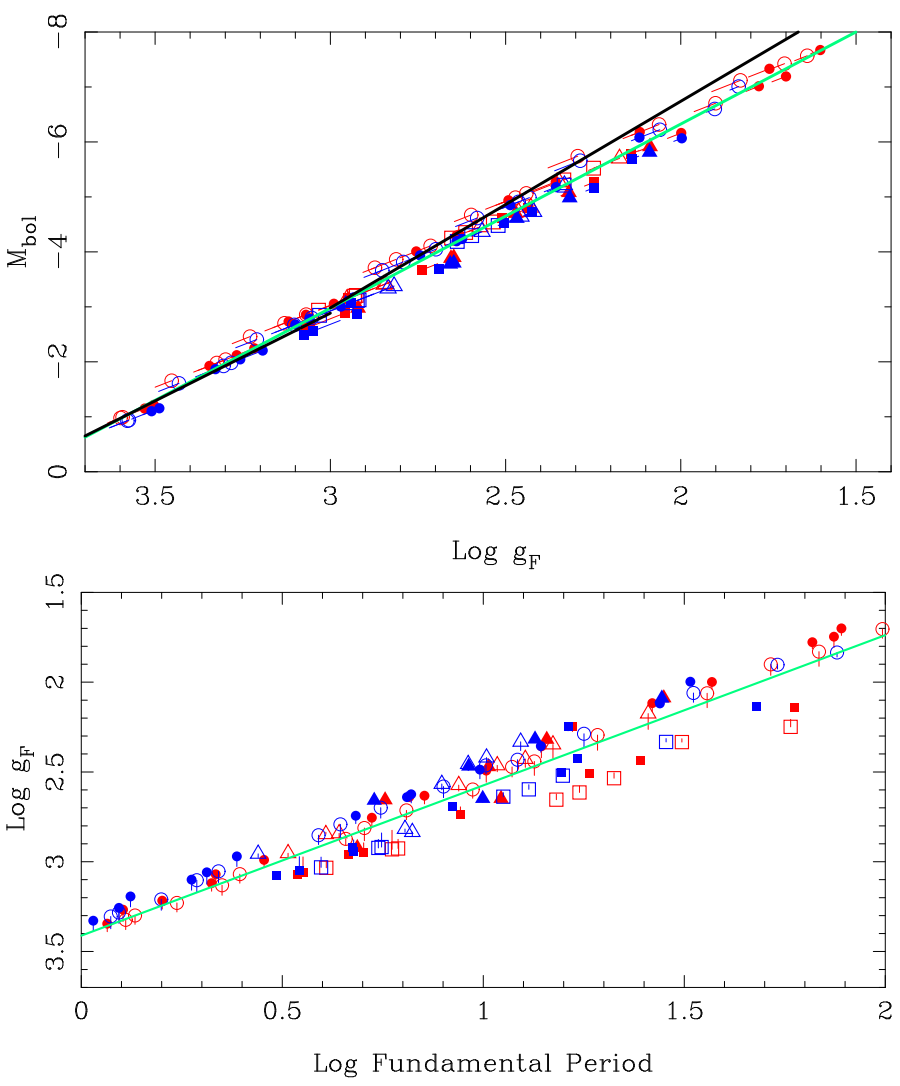

Fig. 1. Top panel: FWGLR based on the pulsation models in Anderson et al. (2016). FU models are shown in red, FO models are shown in blue. For clarity FU (FO) models are plotted with an offset of $+0.05(-0.05)$ dex in $M_{\text {bol }}$. Symbols indicate the entry point of the IS, the lines connect it to the exit point of the IS. The first, second, and third crossing models are plotted as circles, squares, and triangles, respectively. Solar metallicity models are plotted with open symbols, models with $Z=0.006$ with filled symbols. The black lines refer to Eqs. (1) and (2), the green line to the best fit (Eq. (3)). Bottom panel: relation between FWG and period for the same models. The period of the FO models was fundamentalised. The green line refers to the best fit, Eq. (4).

Luck (2018) (hereafter L18) published a list of abundances and stellar parameters for 435 Cepheids based on the analysis of 1137 spectra. L18 reduced all data in a uniform way using MARCS LTE model atmospheres (Gustafsson et al. 2008). Effective temperatures were determined in that paper using the line depth ratio (LDR) - effective temperature calibration of Kovtyukh (2007) as updated by Kovtyukh (2010, priv. comm. to Luck), while gravities were determined from the ionisation balance between Fe I and Fe II lines, and micro-turbulent velocities $\left(v_{\mathrm{t}}\right)$ by forcing there to be no dependence in the per-line Fe I abundances on equivalent width (see L18 for additional details).

Table 1 contains information on the set of $52 \mathrm{CCs}$ for which five or more spectra were available in L18 taken at different phases in the pulsation cycle. FWGs are calculated on the one hand from the mean effective temperatures and mean gravities (as given by L18 in his Table 11), and on the other hand from an analysis of the FWGs calculated for the individual epochs and plotted versus phase. Using the code PERIOD04 (Lenz \& Breger 2005 ) to fit a low-order harmonic, this gives the mean $\log g_{\mathrm{F}}$, the amplitude of the $\log g_{\mathrm{F}}$ curve, and the rms value. Some $\log g_{\mathrm{F}}$ phased curves with fits are shown in Fig. 2. 
M. A. T. Groenewegen: The flux-weighted gravity-luminosity relation of Galactic classical Cepheids

Table 1. FWGL data for the subsample with more than five spectra.

\begin{tabular}{|c|c|c|c|c|c|c|c|c|c|c|}
\hline Name & $\begin{array}{r}\text { Period } \\
\text { (days) }\end{array}$ & $N_{\text {spec }}$ & $\begin{array}{c}\left\langle T_{\text {eff }}\right\rangle \\
(\mathrm{K})\end{array}$ & $\begin{array}{c}\langle\log g\rangle \\
(\mathrm{cgs})\end{array}$ & $\begin{array}{c}\left\langle\log g_{\mathrm{F}}\right\rangle \\
(\mathrm{cgs})\end{array}$ & $N_{\mathrm{h}}$ & $\begin{array}{c}\log g_{\mathrm{F}} \\
(\mathrm{cgs})\end{array}$ & $\begin{array}{l}\text { Ampl } \\
\text { (cgs) }\end{array}$ & $\begin{array}{l}\mathrm{rms} \\
(\mathrm{cgs})\end{array}$ & $\begin{array}{r}\text { Luminosity } \\
\left(L_{\odot}\right)\end{array}$ \\
\hline V473 Lyr & 1.490780 & 5 & 6019 & 2.30 & 3.18 & 1 & 3.205 & 0.056 & 0.006 & $572.3 \pm 10.8$ \\
\hline SU Cas & 1.949324 & 13 & 6274 & 2.26 & 3.07 & 2 & 3.065 & 0.122 & 0.046 & $1027.0 \pm 16.6$ \\
\hline DT Cyg & 2.499215 & 14 & 6192 & 2.27 & 3.10 & 1 & 3.108 & 0.049 & 0.066 & $157.6 \pm 70.7$ \\
\hline SZ Tau & 3.148730 & 16 & 5987 & 2.03 & 2.92 & 1 & 2.937 & 0.079 & 0.081 & $1155.3 \pm 23.1$ \\
\hline V1334 Cyg & 3.332816 & 11 & 6293 & 2.22 & 3.02 & 1 & 3.014 & 0.119 & 0.082 & $1879.5 \pm 384.2$ \\
\hline RT Aur & 3.728485 & 12 & 5948 & 2.06 & 2.96 & 1 & 2.978 & 0.048 & 0.031 & $970.8 \pm 46.1$ \\
\hline SU Cyg & 3.845547 & 12 & 6036 & 2.08 & 2.96 & 1 & 2.988 & 0.067 & 0.136 & $909.6 \pm 294.7$ \\
\hline ST Tau & 4.034299 & 7 & 6052 & 2.07 & 2.94 & 1 & 2.910 & 0.078 & 0.059 & $1155.3 \pm 23.1$ \\
\hline BQ Ser & 4.270900 & 7 & 6040 & 2.16 & 3.04 & 1 & 2.860 & 0.294 & 0.129 & $1876.3 \pm 97.1$ \\
\hline Y Lac & 4.323776 & 10 & 5915 & 1.87 & 2.78 & 1 & 2.779 & 0.111 & 0.108 & $1250.6 \pm 82.7$ \\
\hline T Vul & 4.435462 & 12 & 5852 & 2.03 & 2.96 & 1 & 2.949 & 0.131 & 0.109 & $691.9 \pm 27.6$ \\
\hline FF Aql & 4.470881 & 14 & 6164 & 2.04 & 2.88 & 1 & 2.886 & 0.085 & 0.086 & $2237.6 \pm 118.3$ \\
\hline CF Cas & 4.875220 & 7 & 5672 & 1.74 & 2.73 & 1 & 2.748 & 0.129 & 0.142 & $1284.7 \pm 41.1$ \\
\hline BG Lac & 5.331908 & 9 & 5674 & 1.70 & 2.68 & 2 & 2.710 & 0.218 & 0.035 & $1303.6 \pm 43.2$ \\
\hline delta Cep & 5.366341 & 19 & 5854 & 1.96 & 2.89 & 3 & 2.878 & 0.108 & 0.068 & $1984.8 \pm 587.1$ \\
\hline Y Sgr & 5.773350 & 12 & 5767 & 1.77 & 2.73 & 1 & 2.724 & 0.088 & 0.096 & $1737.4 \pm 90.1$ \\
\hline FM Aql & 6.114290 & 12 & 5766 & 1.68 & 2.64 & 1 & 2.667 & & & $2575.5 \pm 91.8$ \\
\hline X Vul & 6.31 & 8 & & & & 1 & & & & \pm 81.7 \\
\hline XX Sgr & 6.424140 & 5 & 5805 & 1.81 & 2.75 & 1 & 2.671 & & & $=123.3$ \\
\hline AW Per & 6.463589 & 11 & 5928 & 1.86 & 2.77 & 2 & 2.776 & 0.153 & 0.079 & $1646.8 \pm 76.6$ \\
\hline U Sgr & 6.745226 & 11 & 5709 & 1.79 & 2.76 & 2 & 2.769 & 0.178 & 0.086 & $2421.8 \pm 69.6$ \\
\hline U Aql & 7.024049 & 5 & 5565 & 1.64 & 2.66 & 1 & 2.654 & 0.077 & 0.026 & $1619.1 \pm 42.2$ \\
\hline eta Aql & 7.176915 & 14 & 5746 & 1.86 & 2.82 & 1 & 2.793 & 0.095 & 0.124 & $3008.4 \pm 598.2$ \\
\hline BB Her & 7.507945 & 8 & 5641 & 1.65 & 2.64 & 1 & 2.655 & 0.075 & 0.091 & $3122.0 \pm 153.2$ \\
\hline RS Ori & 7.566881 & 7 & 5891 & 1.77 & 2.69 & 1 & 2.693 & 0.109 & 0.112 & $2683.4 \pm 85.8$ \\
\hline V440 Per & 7.570000 & 10 & 6056 & 1.97 & 2.84 & 1 & 2.829 & 0.0 & 0.109 & $3257.4 \pm 50.3$ \\
\hline W Sgr & 7.59 & 9 & & & & 1 & & & & 312.9 \\
\hline RX Cam & 7.912024 & 10 & 5703 & 1.65 & 2.63 & 1 & 2.642 & 0.166 & 0.087 & $2192.7 \pm 87.0$ \\
\hline W Gem & 7.913779 & 13 & 5771 & 1.69 & 2.64 & 1 & 2.662 & 0.131 & 0.074 & $3631.9 \pm 179.0$ \\
\hline U Vul & 7.990676 & 8 & 5779 & 1.73 & 2.68 & 1 & 2.673 & 0.042 & 0.069 & $5408.2 \pm 241.2$ \\
\hline DL Cas & 8.000669 & 11 & 5682 & 1.56 & 2.54 & 2 & 2.566 & & & $1957.5 \pm 49.4$ \\
\hline V636 Cas & 8.375710 & 8 & 5505 & 1.47 & 2.51 & 1 & 2.508 & 0.036 & & $3268.0 \pm 81.6$ \\
\hline S Sge & 8.382086 & 11 & 5689 & 1.73 & 2.71 & 1 & 2.743 & 0.133 & & $2286.1 \pm 57.8$ \\
\hline V500 Sco & 9.316863 & 5 & 5675 & 1.56 & 2.54 & 1 & 2.543 & 0.042 & 0.062 & $2654.7 \pm 143.3$ \\
\hline FN Aql & 9.481640 & 11 & 5488 & 138 & 2.42 & 1 & 2.456 & & & $2699.4 \pm 68.5$ \\
\hline YZ Sgr & 9.553606 & 11 & & & 2. & 1 & & & & 126.8 \\
\hline zeta Gem & 10.150730 & 12 & 5512 & 1.52 & 2.55 & 2 & 2.562 & 0.126 & 0.080 & $3203.4 \pm 928.2$ \\
\hline Z Lac & 10.885613 & 10 & 5618 & 1.49 & 2.49 & 2 & 2.499 & 0.119 & 0.058 & $4173.7 \pm 193.5$ \\
\hline VX Per & 10.889040 & 12 & 5783 & 1.64 & 2.59 & 1 & 2.579 & 0.130 & 0.147 & $4407.1 \pm 107.4$ \\
\hline RX Aur & 11.626000 & 13 & 5782 & 1.67 & 2.62 & 1 & 2.623 & 0.155 & 0.085 & $4670.0 \pm 204.7$ \\
\hline TT Aql & 13.754912 & 10 & 5272 & 1.15 & 2.26 & 2 & 2.400 & 0.402 & 0.104 & $5242.1 \pm 206.0$ \\
\hline SV Mon & 15.232780 & 9 & 5330 & 1.11 & 2.20 & 1 & 2.220 & 0.237 & 0.136 & $4952.3 \pm 352.7$ \\
\hline X Cyg & 16.386332 & 20 & 5252 & 1.10 & 2.22 & 1 & 2.284 & 0.206 & 0.140 & $5201.9 \pm 280.9$ \\
\hline RW Cam & 16.415014 & 17 & 5213 & 1.03 & 2.16 & 1 & 2.200 & 0.100 & 0.156 & $4857.7 \pm 187.4$ \\
\hline CD Cyg & & 17 & & & & 2 & & & 0.103 & $5399.7 \pm 191.6$ \\
\hline Y Oph & 17.124130 & 14 & 5819 & 1.62 & 2.56 & 1 & 2.561 & 0.088 & 0.061 & $12857.9 \pm 388.4$ \\
\hline SZ Aql & 17.141247 & 11 & 5398 & 1.20 & 2.27 & 2 & 2.299 & 0.150 & 0.084 & $7077.7 \pm 232.3$ \\
\hline WZ Sgr & 21.849709 & 10 & 5140 & 0.88 & 2.04 & 2 & 2.204 & 0.514 & 0.060 & $8349.1 \pm 239.9$ \\
\hline X Pup & 25.961000 & 8 & 5353 & 0.75 & 1.84 & 1 & 1.923 & 0.374 & 0.073 & $9419.5 \pm 552.9$ \\
\hline T Mon & 27.024649 & 12 & 5108 & 0.93 & 2.10 & 1 & 2.115 & & 0.141 & $8163.2 \pm 203.3$ \\
\hline SV Vul & 45.012100 & 15 & 5329 & 0.85 & 1.94 & 1 & 1.905 & 0.124 & 0.157 & $27925.1 \pm 1818.3$ \\
\hline S Vul & 68.463997 & 6 & 5452 & 0.93 & 1.98 & 1 & 1.929 & 0.281 & 0.082 & $21197.2 \pm 747.9$ \\
\hline
\end{tabular}

Notes. Column 1: Name. Column 2: Period (as quoted in L18). Column 3: Number of spectra L18. Column 4: Average effective temperature (quoted in Table 11 in L18). Column 5: Average $\log g$ (quoted in Table 11 in L18). Column 6: Average $\log g_{\mathrm{F}}$ based on Cols. 4 and 5. Column 7: Number of harmonics used in the time analysis. Column 8: Mean $\log g_{\mathrm{F}}$. Column 9: Amplitude in the $\log g_{\mathrm{F}}$ curve. Column 10: rms. Column 11: Luminosity and error (from Table 1 in G20). The error is the fit error, and does not include the error on the distance. The distance and error on the distance needed to calculate the total error on $L$ are given in Table A.3. 

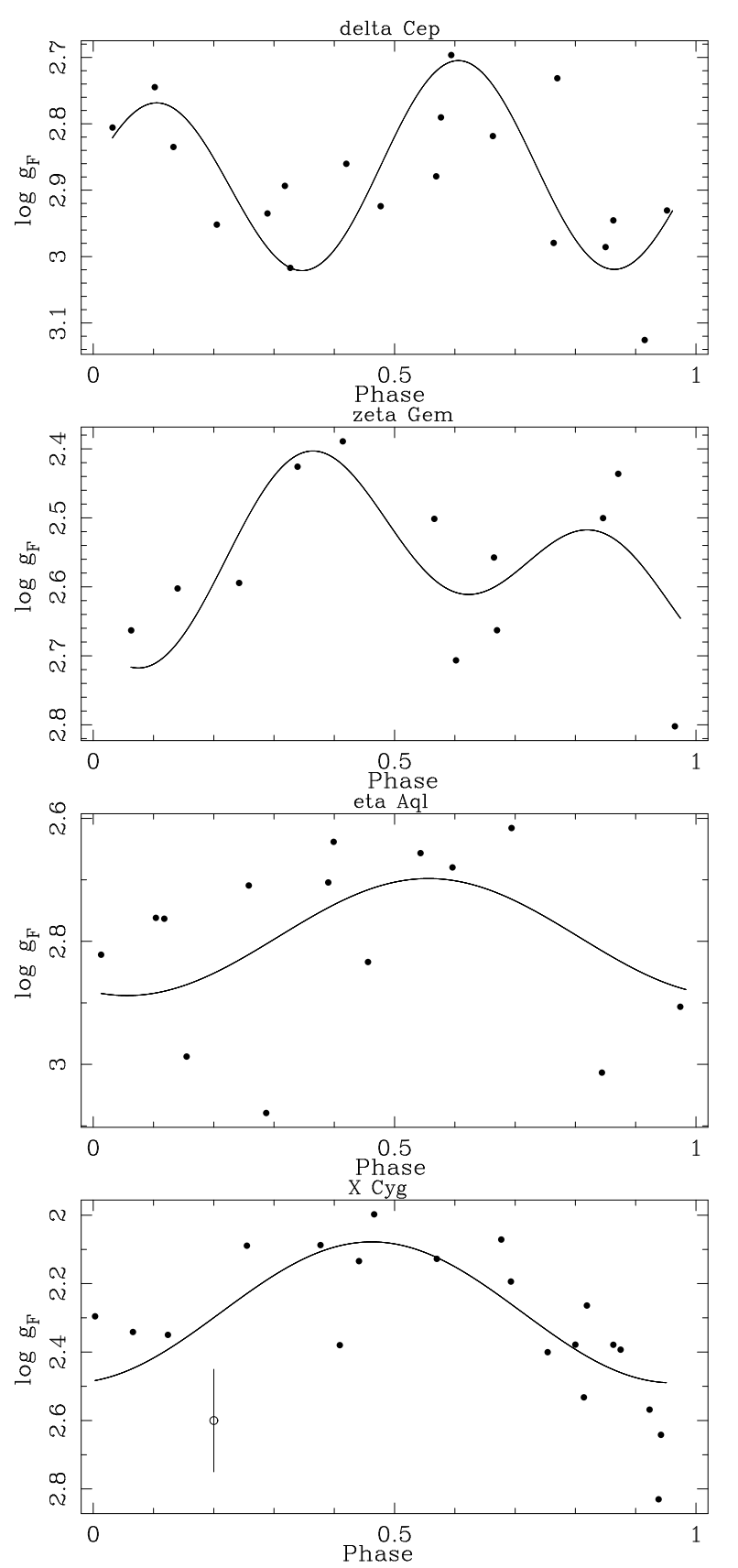

Fig. 2. FWG vs pulsation phase for four CCs. The typical error bar in each point is 0.15 dex in FWG, as indicated in the bottom plot. The lines are low-order harmonic fits to the data (see Col. 7 in Table 1).

These curves show considerable scatter even when the pulsation cycle is well sampled. This is likely due to the error bar in an individual determination of $g_{\mathrm{F}}$. The error on effective temperature generally has a negligible contribution in this. Ninety-five percent of individual effective temperature error bars among the 1137 spectra in L18 are between 30 and $220 \mathrm{~K}$ with a median of $65 \mathrm{~K}$. An error of $100 \mathrm{~K}$ at $T_{\text {eff }}=6000 \mathrm{~K}$ introduces an error of $0.03 \mathrm{dex}$ in $g_{\mathrm{F}}$, much smaller than the error on $\log g$, which was estimated to be $\sim 0.15$ dex by L18. A comparison of $\log g_{\mathrm{F}}$ values determined from the averages of the effective temperatures and gravities, and from fitting the $\log g_{\mathrm{F}}$ curve with phase show essentially the same result, especially when seven of more spectra are averaged (with an average difference between Cols. 6 and 8 of $-0.02 \pm 0.04$ dex).

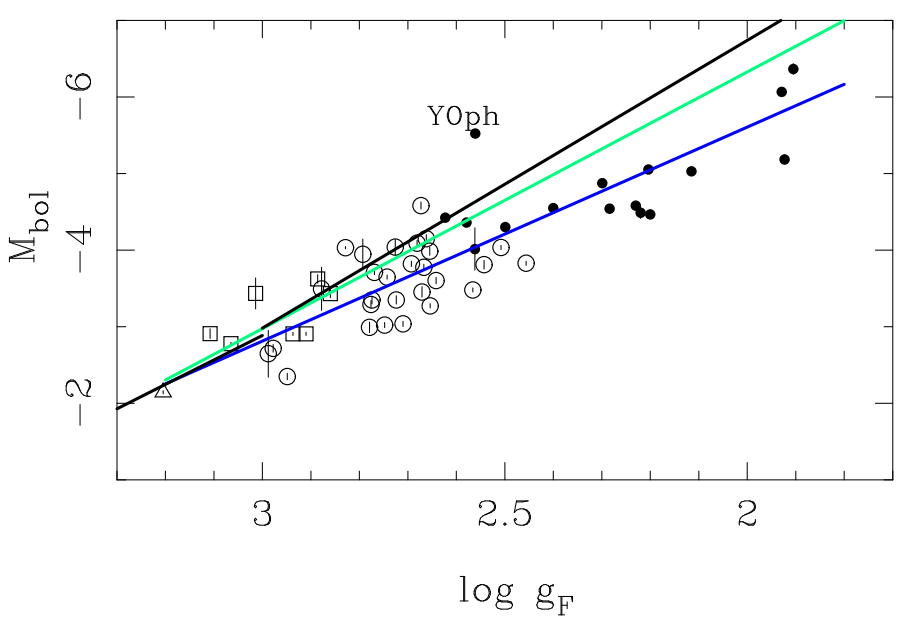

Fig. 3. FWGLR based on the subsample with more than five spectra. FU mode pulsators are plotted as circles (filled circles for periods over 10 days), FO pulsators as open squares, the single second-overtone pulsator as open triangle. The black lines refer to Eqs. (1) and (2), the green line to Eq. (3). The blue line is a fit to the data points, excluding Y Oph (Eq. (5)).

Figure 3 shows the FWGLR for the sample of 52 stars from Table 1, where the luminosity and error are taken from G20. Equations (1)-(3) are plotted as reference. Using a linear bisector fit (using the code SIXLIN from Isobe et al. 1990) the best fit is

$M_{\text {bol }}=(2.79 \pm 0.18)\left(\log g_{\mathrm{F}}-2.5\right)+(-4.21 \pm 0.08)$

with an rms of 0.38 mag (blue line in the figure). A standard least-squares fit has a shallower slope of 2.54. The theoretical fit is shown in Eq. (3), and this fit differs by about $0.4 \mathrm{mag}$ at $\log g_{\mathrm{F}}=2.5$. Alternatively, the observed $\log g_{\mathrm{F}}$ values are systematically too small by $0.4 / 2.8 \sim 0.14$ dex. At lower FWG or longer periods the difference with the theoretical relation is larger.

Table A. 3 collects the FWG data for the entire sample of 477 stars. Overall, most of the data (435 stars) come from L18, and for the remaining stars $\log g$ and $T_{\text {eff }}$ have been collected from the literature in order to calculate $\log g_{\mathrm{F}}$. Multiple determinations of $\log g_{\mathrm{F}}$ have been averaged and so can differ slightly from the values in Table 1. The table also includes the period, pulsation type, distance with error, and luminosity with error from G20. Figure 4 shows the observational equivalent to the bottom panel in Fig. 1, the FWG determined from spectroscopy against pulsation period (fundamentalised for FO pulsators).

There is a tight correlation between the two quantities. Removing non-CCs (see Table A.3) and applying iterative $3 \sigma$ clipping results in the fit

$\log g_{\mathrm{F}}=(-0.80 \pm 0.03) \log P_{0}+(3.43 \pm 0.03)$

with an rms of 0.16 dex, in very good agreement with the theoretically predicted relation. Interestingly, many of the outliers come from a single source, Genovali et al. (2014), who derived very $\operatorname{low} \log g$ values for some objects. Some additional information and fits are provided in Appendix A.

Figure 5 is the equivalent to Fig. 3 for the entire sample, using a simple averaging of the available FWGs. The error on distance is now taken into account in calculating the error on luminosity. Following the discussion above and in the appendix, the data from Genovali et al. (2014) has been excluded, and to 


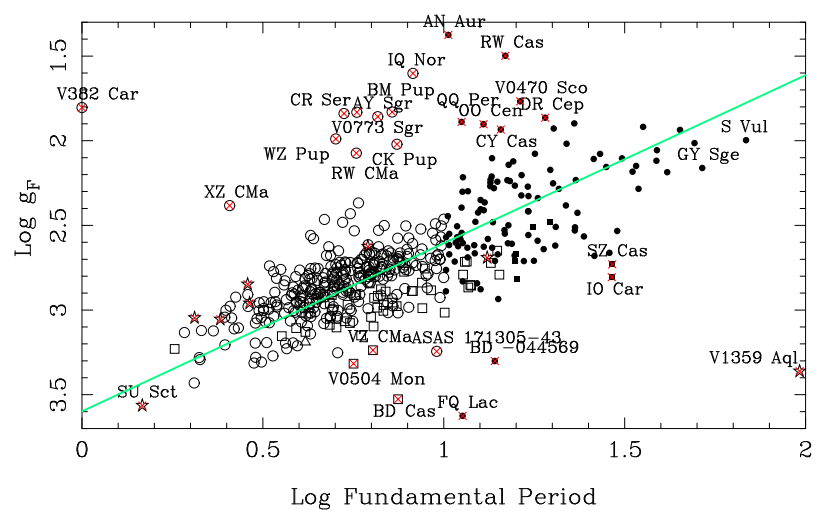

Fig. 4. FWG vs fundamental pulsation period. Some outliers are named. The green line refers to the best fit, Eq. (7), which excludes the outliers and non-CCs indicated by a red cross.

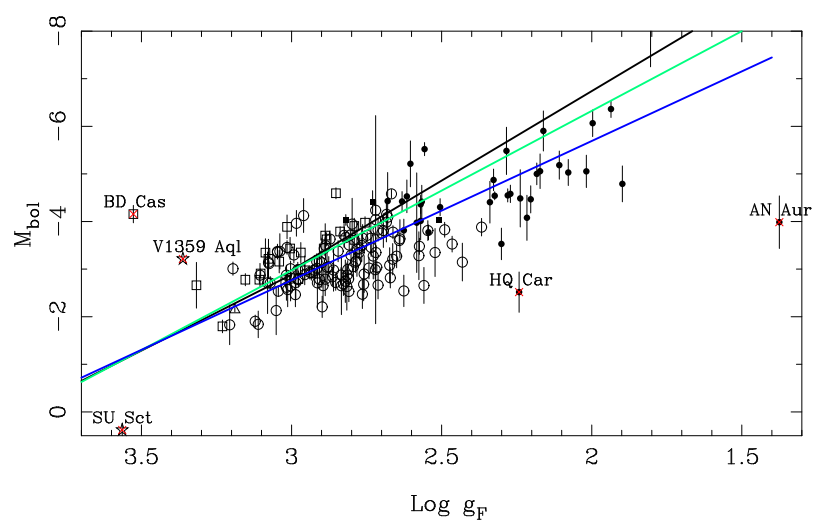

Fig. 5. FWGLR, with some outliers named. The black lines refer to Eqs. (1) and (2), the green line to Eq. (3). The blue line refers to the best fit, Eq. (7), which excludes the outliers and non-CCs indicated by a red cross. Outliers located outside the plot window are SU Cru $\left(\log g_{\mathrm{F}}=\right.$ $\left.0.19, M_{\mathrm{bol}}=-3.7\right)$, SY Nor $\left(\log g_{\mathrm{F}}=2.4, M_{\mathrm{bol}}=+3.3\right)$, and V382 Car $\left(\log g_{\mathrm{F}}=1.8, M_{\mathrm{bol}}=-8.6\right)$.

reduce the scatter only stars with two or more spectra are considered. A linear bi-sector fit applying iterative $3 \sigma$ clipping results in

$M_{\mathrm{bol}}=(2.93 \pm 0.13)\left(\log g_{\mathrm{F}}-2.5\right)+(-4.23 \pm 0.06)$

with an rms of 0.54 mag using 170 stars and is shown as the blue line in the figure. This is currently the best observational determination of the FWGL relation for CCs.

\section{Discussion and summary}

The relation between FWG and period, and FWG and bolometric luminosity is investigated for a sample $477 \mathrm{CCs}$. The FWGs are derived from effective temperatures and $\log g$ values available in the literature based on high-resolution spectroscopy. The overall majority of parameters have been compiled from a single source (L18) that determined $\log g$ and $T_{\text {eff }}$ in an uniform manner. For a subset of stars multiple-phase data is available. The FWG-Period and FWGLR are compared to theoretical models from Anderson et al. (2016)

A very good agreement is found between the theoretical and observed relations between FWG and period. These relations could serve as a prediction for a reasonable range in $\log g$ values (assuming an effective temperature) in a spectroscopic analysis.
The observed FWGLR is found to have a shallower slope than the theoretical relation. It is not clear at the moment if this is a significant effect or not. As the observed relation between FWG and period agrees with the theoretical relation, one would be inclined to think that there could be a systematic effect in the bolometric magnitudes of the long-period Cepheids. They are rarer and on average at longer distance, likely to be more susceptible to (systematic) errors on parallax. This is qualitatively confirmed by repeating the fit of Eq. (7) restricting the sample to stars with $\sigma_{\mathrm{L}} / L<0.2$. The slope is increased, but has a larger error bar $(3.05 \pm 0.19)$ and the rms is reduced to 0.44 mag.

On the other hand, although the $T_{\text {eff }}$ determinations based on the LDR method are precise (as discussed earlier), possible systematic effects (which would also affect the determination of $\log g$ and $\log g_{\mathrm{F}}$ ) could play a role (Mancino et al. 2020). For the subsample of 52 stars in L18 with five or more spectra, the cycle averaged $T_{\text {eff }} \mathrm{s}$ (as quoted in Table 1) are compared to the photometrically derived $T_{\mathrm{eff}} \mathrm{s}$ based on the SED fitting in G20. The errors on the photometrically derived effective temperatures (the median is $180 \mathrm{~K}$ ) are larger than those derived from spectroscopy. There are two outliers Y Oph and S Vul, where the photometrically derived temperatures are considerably lower than those quoted in L18 $(570$ and $830 \mathrm{~K} ;>4.3 \sigma)$. For the other stars the difference (spectroscopically - photometrically derived $\left.T_{\text {eff }}\right)$ is $140 \pm 150 \mathrm{~K}$.

Systematic errors on the determination of the gravity could also play a role. The methodology used by L18 to determine the stellar parameters, in particular $v_{\mathrm{t}}$ and gravity, is the standard one. A non-standard method is sometimes also used in the literature, as introduced by Kovtyukh \& Andrievsky (1999). To avoid non-LTE-sensitive stronger Fe I lines, $v_{\mathrm{t}}$ is derived from Fe II lines and weak Fe I lines alone. This leads to higher $v_{\mathrm{t}}$, which in turn leads to higher gravities when the ionisation balance is enforced. For $\delta$ Cep Kovtyukh \& Andrievsky (1999) find that the gravities are higher by 0.5 dex using the non-standard method. The matter is also debated in Yong et al. (2006). They note that the non-standard method "has merits", but show that their derived gravities using the standard method are self-consistent, one argument being that this gravity also produces ionisation equilibrium for Ti I lines that are more susceptible to non-LTE effects than Fe. The non-standard method is also used in Takeda et al. (2013). Anderson et al. (2016) excluded the gravities from that paper as they differed from other sources they used. Twelve stars overlap with the sample of stars with multiepoch data from L18 (in Table 1). Takeda et al. (2013) present stellar parameters at between 7 and 17 epochs. The mean effective temperatures and mean gravities are calculated, as well are FWGs at these epochs based on the data in Takeda et al. (2013), and fitted with low-order harmonic sine curves, as before, to give the mean FWG. The difference (min-max (mean)) between the parameters from the non-standard method minus those from the standard method are $-8-+444(167) \mathrm{K}$ in $T_{\text {eff }},+0.22-$ $+0.72(+0.36)$ dex in $\log g$, and $+0.11-+0.67(+0.34)$ dex in FWG, with tendencies that the difference in all three quantities decreases with increasing period.

The FWGLR has the potential to be an alternative to the $P L$ relation in distance determination (Anderson et al. 2016). In its current empirically best calibrated version it is not. The scatter of $0.54 \mathrm{mag}$ is larger than the $0.40 \mathrm{mag}$ in the bolometric $P L$ relation determined in G20 using the identical sample of stars, distances, and luminosities.

One issue is that the independent variable period is known with great precision, while the independent variable FWG has a non-negligible error associated with it. The fitting of the FWG 
versus pulsation phase did not provide more precise mean FWGs than simple averaging. As the slope of the FWGLR is reasonably steep, any uncertainty on the FWG leads to a three times larger uncertainty in $M_{\text {bol }}$.

The discussion above also demonstrates that the stellar parameters should be derived in a uniform way. To exclude the influence of data analysis inhomogeneity altogether, Eq. (7) was re-determined using data only from L18. The usable sample is reduced to 161 stars and the slope and offset change marginally, less than $1 \sigma$. The standard approach used by L18 seems to give consistent results when considering the comparison to theory and the independent calibration of the FWGLR by Kudritzki et al. (2020). Changes in the FWG by $\sim+0.3-0.5$ dex, as implied by the non-standard method, would result in a disagreement.

This paper is written with the tremendous potential offered by Gaia in mind. Future data releases will provide information that will impact and improve on the results obtained here. Primarily, improved parallaxes, taking into account binarity in the astrometrical solution, will provide more precise distances and thus bolometric luminosities (e.g. through the SED fitting performed in G20).

Secondly, Gaia RVS spectra and Gaia BP/RP spectrophotometry will provide estimates of the stellar parameters $\left(\log g, T_{\text {eff }}\right.$, also metallicity) in future releases. Only mean spectra in data release 3, and epoch spectra in data release 4 (Brown 2019). An older analysis by Recio-Blanco et al. (2016) indicate that end-of-mission accuracies in $\log g$ of $0.1 \mathrm{dex}$ or better can be reached in intermediate-metallicity $\mathrm{F}$ and $\mathrm{G}$ giants of magnitude $G \sim 10.3-11.8$ or brighter. Spectro-photometry can go fainter but with poorer accuracies $(0.2-0.4$ dex in $\log g$ down to $G=19$; Table 4 in Bailer-Jones et al. 2013). As the nominal mission of 5 years is extended, by +18 months until the end of 2020 , and likely until the end of 2022, these numbers should improve. In conclusion, the FWGLR could prove to become an extremely useful tool in Cepheid studies.

Acknowledgements. I would like to thank Dr. Bertrand Lemasle for interesting discussion on the determination of $\log g$ and commenting on a draft version of this paper. This research has made use of the SIMBAD database and the VizieR catalogue access tool operated at CDS, Strasbourg, France.

\section{References}

Anders, F., Khalatyan, A., Chiappini, C., et al. 2019, A\&A, 628, A94
Anderson, R. I., Saio, H., Ekström, S., Georgy, C., \& Meynet, G. 2016, A\&A, 591, A8

Anderson, R. I., Saio, H., Ekström, S., Georgy, C., \& Meynet, G. 2020, A\&A, 638, C1

Andrievsky, S. M., Kovtyukh, V. V., \& Usenko, I. A. 1994, A\&A, 281, 465

Andrievsky, S. M., Kovtyukh, V. V., Luck, R. E., et al. 2002a, A\&A, 392, 491

Andrievsky, S. M., Kovtyukh, V. V., Luck, R. E., et al. 2002b, A\&A, 381, 32

Andrievsky, S. M., Luck, R. E., Martin, P., \& Lépine, J. R. D. 2004, A\&A, 413, 159

Andrievsky, S. M., Lépine, J. R. D., Korotin, S. A., et al. 2013, MNRAS, 428, 3252

Andrievsky, S. M., Martin, R. P., Kovtyukh, V. V., Korotin, S. A., \& Lépine, J. R. D. 2016, MNRAS, 461, 4256

Bailer-Jones, C. A. L., Andrae, R., Arcay, B., et al. 2013, A\&A, 559, A74

Boyarchuk, A. A., \& Lyubimkov, L. S. 1981, Bull. Crim. Astrophys. Obs., 64, 1

Brown, A. G. A. 2019, https://doi .org/10.5281/zenodo. 2637972

Feast, M. W., \& Catchpole, R. M. 1997, MNRAS, 286, L1

Gaia Collaboration (Brown, A. G. A., et al.) 2018, A\&A, 616, A1

Genovali, K., Lemasle, B., Bono, G., et al. 2014, A\&A, 566, A37

Groenewegen, M. A. T. 2018, A\&A, 619, A8

Groenewegen, M. A. T. 2020, A\&A, 635, A33

Gustafsson, B., Edvardsson, B., Eriksson, K., et al. 2008, A\&A, 486, 951

Inno, L., Urbaneja, M. A., Matsunaga, N., et al. 2019, MNRAS, 482, 83

Isobe, T., Feigelson, E. D., Akritas, M. G., \& Babu, G. J. 1990, ApJ, 364, 104

Kovtyukh, V. V. 2007, MNRAS, 378, 617

Kovtyukh, V. V., \& Andrievsky, S. M. 1999, A\&A, 351, 597

Kovtyukh, V. V., Wallerstein, G., \& Andrievsky, S. M. 2005, PASP, 117, 1173

Kudritzki, R. P., Bresolin, F., \& Przybilla, N. 2003, ApJ, 582, L83

Kudritzki, R.-P., Urbaneja, M. A., Bresolin, F., et al. 2008, ApJ, 681, 269

Kudritzki, R. P., Castro, N., Urbaneja, M. A., et al. 2016, ApJ, 829, 70

Kudritzki, R.-P., Urbaneja, M. A., \& Rix, H.-W. 2020, ApJ, 890, 28

Lemasle, B., François, P., Bono, G., et al. 2007, A\&A, 467, 283

Lemasle, B., François, P., Piersimoni, A., et al. 2008, A\&A, 490, 613

Lemasle, B., Kovtyukh, V., Bono, G., et al. 2015, A\&A, 579, A47

Lenz, P., \& Breger, M. 2005, Commun. Asteroseismol., 146, 53

Luck, R. E. 2018, AJ, 156, 171

Luck, R. E., Gieren, W. P., Andrievsky, S. M., et al. 2003, A\&A, 401, 939

Luck, R. E., Kovtyukh, V. V., \& Andrievsky, S. M. 2006, AJ, 132, 902

Mancino, S., Romaniello, M., Anderson, R. I., \& Kudritzki, R. P. 2020, ArXiv e-prints [arXiv:2001.05881]

Martin, R. P., Andrievsky, S. M., Kovtyukh, V. V., et al. 2015, MNRAS, 449, 4071

Pietrzyński, G., Graczyk, D., Gallenne, A., et al. 2019, Nature, 567, 200

Recio-Blanco, A., de Laverny, P., Allende Prieto, C., et al. 2016, A\&A, 585, A93

Riess, A. G., Casertano, S., Yuan, W., et al. 2018, ApJ, 861, 126

Riess, A. G., Casertano, S., Yuan, W., Macri, L. M., \& Scolnic, D. 2019, ApJ, 876,85

Romaniello, M., Primas, F., Mottini, M., et al. 2008, A\&A, 488, 731

Schmidt, E. G., Rogalla, D., \& Thacker-Lynn, L. 2011, AJ, 141, 53

Takeda, Y., Kang, D. I., Han, I., Lee, B. C., \& Kim, K. M. 2013, MNRAS, 432, 769

Watson, C. L., Henden, A. A., \& Price, A. 2006, Soc. Astron. Sci. Annu. Symp., 25,47

Yong, D., Carney, B. W., Teixera de Almeida, M. L., \& Pohl, B. L. 2006, AJ, 131, 2256 
M. A. T. Groenewegen: The flux-weighted gravity-luminosity relation of Galactic classical Cepheids

\section{Appendix A: Additional material}

Table A.1. Fits of the type $M_{\mathrm{bol}}=a \cdot\left(\log g-g_{\mathrm{F}}^{\mathrm{b}}\right)+b$.

\begin{tabular}{lccc}
\hline \hline$a$ & $b$ & rms & Metallicity \\
\hline $3.381 \pm 0.025$ & $-3.031 \pm 0.014$ & 0.13 & $Z=0.014$ \\
3.35 fixed & $-3.040 \pm 0.014$ & 0.14 & \\
$3.331 \pm 0.029$ & $-2.912 \pm 0.017$ & 0.16 & $Z=0.006$ \\
3.35 fixed & $-2.905 \pm 0.014$ & 0.16 & \\
$3.426 \pm 0.026$ & $-2.732 \pm 0.016$ & 0.17 & $Z=0.002$ \\
3.35 fixed & $-2.759 \pm 0.012$ & 0.18 & \\
\hline
\end{tabular}

Additional fits for the FWGLR based on the models of Anderson et al. (2016) are given in Table A.1 for the three different metallicities, and with the slope fixed to the value in Eq. (3). The results for $Z=0.006$ and 0.014 agree within the error and justify the use of a single relation combining the two metallicities (Eq. (3)). The $Z=0.002$ models differ by a larger amount, qualitatively in agreement with the remark in Kudritzki et al. (2020) on the fact that low metallicities (below -0.6 dex) have an effect on the FWGLR.

The bottom panel of Fig. 1 and Eq. (4) present the relation between FWG and pulsation period based on the models of Anderson et al. (2016) with the overtone periods converted to FU periods. Figure A.1 and Eqs. (A.1) and (A.2) give the results for FU and FO pulsators separately. The best fits are

$\log g_{\mathrm{F}}=(-0.847 \pm 0.015) \log P+(3.431 \pm 0.016)$

with an rms of 0.10 dex for the FU models, and

$\log g_{\mathrm{F}}=(-0.840 \pm 0.016) \log P+(3.255 \pm 0.013)$

with an rms of 0.08 dex for the FO models.

Additional fits for the relations between FWG and period are given in Table A.2 and are illustrated in Fig. A.2. They show that when multiple $g_{\mathrm{F}}$ values are available the scatter in the relation decreases. Assuming that the intrinsic scatter in the relation is 0.093 dex (Eq. (4)) a single determination has an estimated error of about 0.13 dex (dominated by the error on $\log g$ ), while averaging six or more spectra leads to an error of about 0.09 dex.

As noted in the main text, and illustrated by comparing Fig. 4 and the top panel in Fig. A.2, a fair fraction of
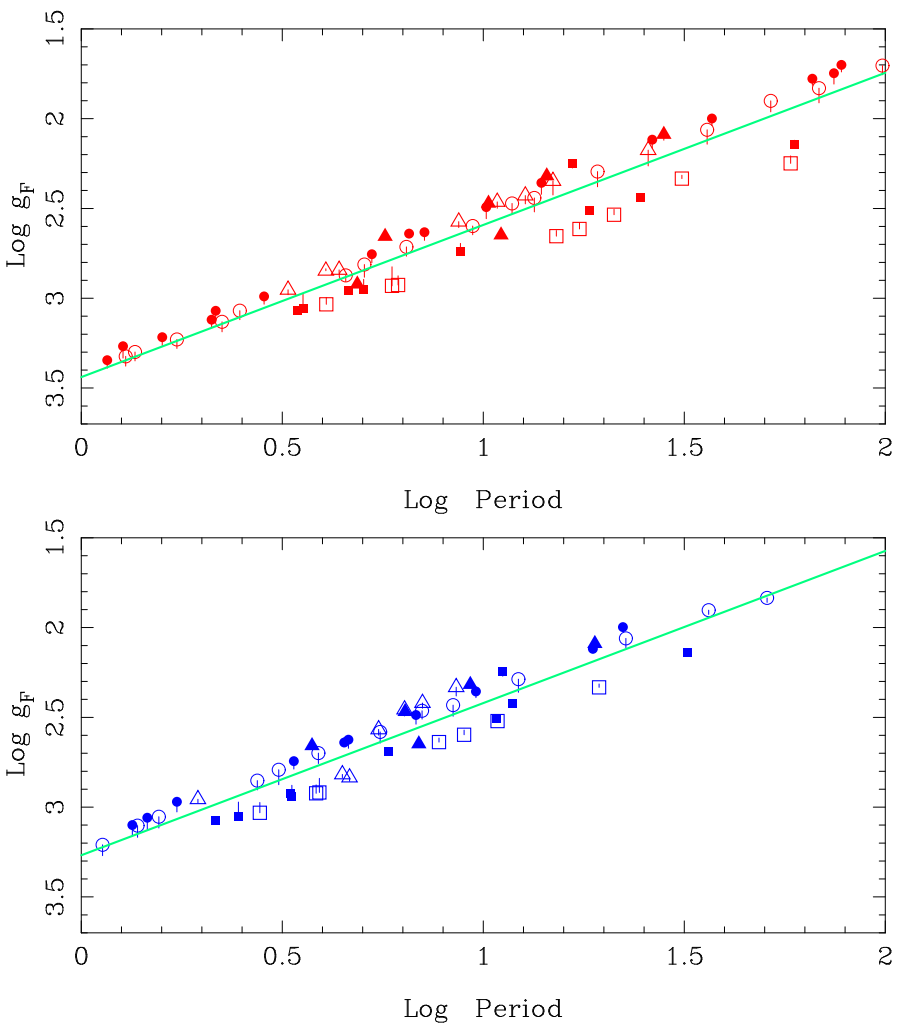

Fig. A.1. Relation between FWG and period for FU (top panel) and FO (bottom panel) models. The meaning of the symbols and colours is explained in Fig 1. The green lines refer to the best fits, Eqs. (A.1) and (A.2).

outliers are stars with $T_{\text {eff }}$ and $\log g$ taken from Genovali et al. (2014). Genovali et al. (2014) also present multiple observations for some stars, and XX Sgr and WZ Sgr are in common with the subsample of stars in L18 with five or more available spectra. A comparison shows that the difference in $\log g_{\mathrm{F}}$ is dominated by the difference in $\log g$, that are of the order 0.5 dex. For some of the stars in the present sample the $\log g_{\mathrm{F}}$ (and $\log g$ ) values are too low by 1 dex. As they seem to use the same methodology as L18 in deriving the stellar parameters, no simple explanation is offered to explain this discrepancy.

Table A.3 compiles the FWG and luminosity data for the entire sample. The full table is available at the CDS. 
Table A.2. Fits of the type $g_{\mathrm{F}}=a \cdot \log P+b$.

\begin{tabular}{lcrrl}
\hline \hline$a$ & $b$ & $\mathrm{rms}$ & $\mathrm{N}$ & Remarks \\
\hline$-0.802 \pm 0.028$ & $3.436 \pm 0.025$ & 0.159 & 443 & Standard, Eq. (7) \\
$-0.804 \pm 0.028$ & $3.438 \pm 0.025$ & 0.158 & 442 & Excluding Genovali et al. (2014) \\
$-0.793 \pm 0.036$ & $3.438 \pm 0.032$ & 0.160 & 275 & $N_{\text {sp }}=1$, excluding Genovali et al. (2014) \\
$-0.752 \pm 0.073$ & $3.381 \pm 0.060$ & 0.172 & 87 & $N_{\text {sp }}=2$, excluding Genovali et al. (2014) \\
$-0.630 \pm 0.116$ & $3.351 \pm 0.100$ & 0.160 & 32 & $N_{\text {sp }}=3-5$, excluding Genovali et al. (2014) \\
$-0.805 \pm 0.106$ & $3.370 \pm 0.110$ & 0.139 & 20 & $N_{\text {sp }}=6-10$, excluding Genovali et al. (2014) \\
$-1.046 \pm 0.088$ & $3.663 \pm 0.086$ & 0.125 & 31 & $N_{\text {sp }} \geq 11$, excluding Genovali et al. (2014) \\
$-0.970 \pm 0.063$ & $3.560 \pm 0.064$ & 0.127 & 50 & $N_{\text {sp }} \geq 6$, excluding Genovali et al. (2014) \\
\hline
\end{tabular}
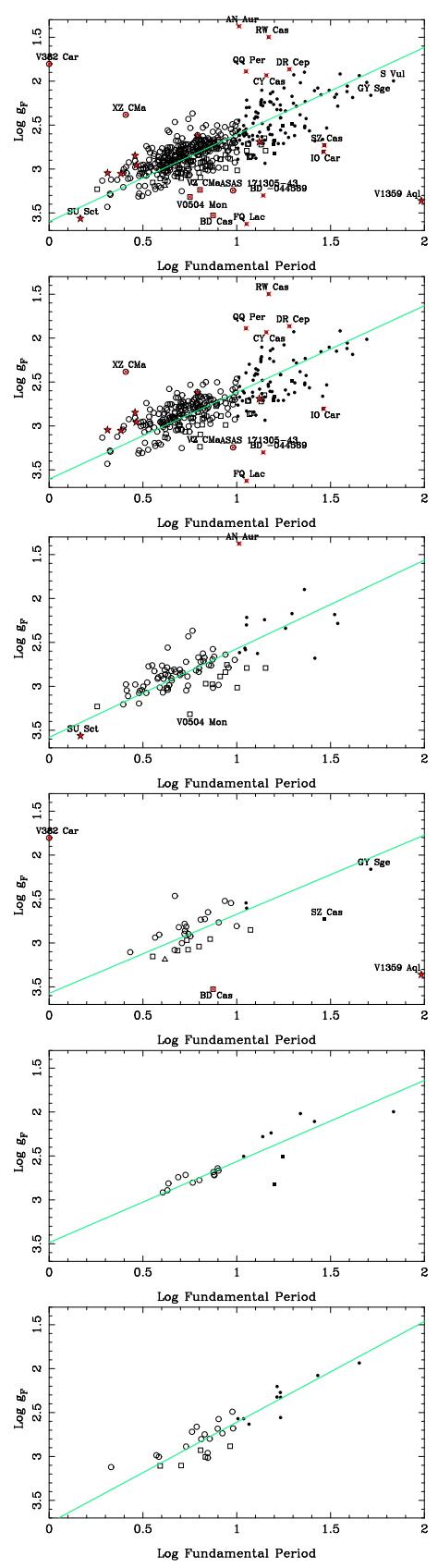

Fig. A.2. FWG vs period. The data from Genovali et al. (2014) is excluded in all plots. Different panels show different selections on the number of available $g_{\mathrm{F}}$ values. From top to bottom: all, $N_{\mathrm{sp}}=1, N_{\mathrm{sp}}=2$, $N_{\mathrm{sp}}=3-5, N_{\mathrm{sp}}=6-10, N_{\mathrm{sp}} \geq 11$. The green lines refer to the best fits (see Table A.2). 
M. A. T. Groenewegen: The flux-weighted gravity-luminosity relation of Galactic classical Cepheids

Table A.3. FWG data for the entire sample (first entries only).

\begin{tabular}{|c|c|c|c|c|c|c|c|c|c|c|c|}
\hline Name & Type & $\begin{array}{r}\text { Period } \\
\text { (days) }\end{array}$ & $\begin{array}{c}d \\
(\mathrm{kpc})\end{array}$ & $\begin{array}{c}\sigma_{\mathrm{d}} \\
(\mathrm{kpc})\end{array}$ & $\begin{array}{r}L \\
\left(L_{\odot}\right) \\
\end{array}$ & $\begin{array}{r}\sigma_{\mathrm{L}} \\
\left(L_{\odot}\right) \\
\end{array}$ & $N_{\text {spec }}$ & $\begin{array}{c}\log g_{\mathrm{F}} \\
(\mathrm{cgs})\end{array}$ & $\begin{array}{c}\sigma_{\log g_{\mathrm{F}}} \\
(\mathrm{cgs})\end{array}$ & $\begin{array}{c}\text { Min-Max } \\
\text { (cgs) }\end{array}$ & Ref. \\
\hline AA Gem & DCEP & 11.302 & 3.400 & 0.829 & 3400.0 & 122.7 & 2 & 2.216 & 0.11 & 0.04 & 1 \\
\hline AA Mon & DCEP & 3.938 & 3.922 & 0.709 & 922.8 & 33.6 & 1 & 3.211 & 0.16 & - & 1 \\
\hline AB Cam & DCEP & 5.788 & 4.200 & 0.966 & 1463.5 & 79.3 & 1 & 2.754 & 0.15 & - & 1 \\
\hline AC Mon & DCEP & 8.014 & 2.400 & 0.400 & 1991.6 & 42.2 & 4 & 2.766 & 0.08 & 0.21 & 1 \\
\hline AD Cam & DCEP & 11.261 & 4.600 & 0.756 & 2048.8 & 87.0 & 2 & 2.301 & 0.11 & 0.07 & 1 \\
\hline AD Cru & DCEP & 6.398 & 2.994 & 0.394 & 1881.9 & 93.2 & 1 & 2.730 & 0.15 & - & 1 \\
\hline AD Gem & DCEP & 3.788 & 2.500 & 0.673 & 966.0 & 32.0 & 2 & 2.914 & 0.11 & 0.12 & 1 \\
\hline AD Pup & DCEP & 13.596 & 4.100 & 0.946 & 4650.8 & 356.8 & 1 & 2.103 & 0.15 & - & 1 \\
\hline AE Tau & DCEP & 3.897 & 3.367 & 0.606 & 953.2 & 11.9 & 1 & 2.802 & 0.15 & - & 1 \\
\hline AE Vel & DCEP & 7.134 & 2.100 & 0.187 & 1842.6 & 169.2 & 1 & 2.663 & 0.15 & - & 1 \\
\hline AG Cru & DCEP & 3.837 & 1.506 & 0.094 & 1773.5 & 49.7 & 1 & 2.864 & 0.15 & - & 1 \\
\hline AH Vel & DCEPS & 4.227 & 0.752 & 0.035 & 2604.0 & 37.7 & 2 & 2.838 & 0.11 & 0.04 & 1 \\
\hline alpha UMi & DCEPS & 3.970 & 0.133 & 0.002 & 2410.9 & 622.8 & 2 & 2.888 & 0.11 & 0.14 & 2,3 \\
\hline AN Aur & DCEP & 10.291 & 3.400 & 0.985 & 3124.5 & 58.2 & 2 & 2.630 & 0.11 & 0.24 & 1 \\
\hline AO Aur & DCEP & 6.763 & 3.400 & 0.839 & 1620.9 & 49.3 & 3 & 2.728 & 0.09 & 0.18 & 1 \\
\hline $\mathrm{AO} \mathrm{CMa}$ & DCEP & 5.816 & 3.600 & 0.434 & 1197.9 & 58.1 & 1 & 2.950 & 0.16 & - & 1 \\
\hline AP Pup & DCEP & 5.084 & 1.183 & 0.051 & 2579.5 & 87.4 & 1 & 2.869 & 0.15 & - & 1 \\
\hline AP Sgr & DCEP & 5.058 & 0.861 & 0.041 & 1651.8 & 38.7 & 1 & 2.780 & 0.15 & - & 1 \\
\hline AQ Car & DCEP & 9.769 & 3.030 & 0.317 & 3837.4 & 289.0 & 1 & 2.702 & 0.15 & - & 1 \\
\hline AQ Pup & DCEP & 30.149 & 2.900 & 0.336 & 11481.5 & 330.8 & 1 & 2.533 & 0.15 & - & 1 \\
\hline AS Per & DCEP & 4.973 & 1.200 & 0.087 & 1042.0 & 36.7 & 2 & 2.810 & 0.11 & 0.30 & 1 \\
\hline AT Pup & DCEP & 6.665 & 1.637 & 0.085 & 2495.6 & 194.9 & 1 & 2.757 & 0.15 & - & 1 \\
\hline AV Cir & DCEPS & 3.065 & 0.944 & 0.033 & 2169.7 & 85.7 & 1 & 2.843 & 0.15 & - & 1 \\
\hline AV Sgr & DCEP & 15.415 & 2.100 & 0.287 & 4413.1 & 139.5 & 1 & 2.609 & 0.15 & - & 1 \\
\hline AW Per & DCEP & 6.464 & 0.700 & 0.044 & 1646.8 & 76.6 & 11 & 2.802 & 0.05 & 0.47 & 1 \\
\hline AX Cir & DCEP & 5.273 & 0.500 & 0.151 & 1854.6 & 33.1 & 3 & 2.782 & 0.09 & 0.08 & 1 \\
\hline AX Vel & $\operatorname{DCEP}(\mathrm{B})$ & 2.593 & 1.517 & 0.077 & 1750.2 & 166.6 & 2 & 3.047 & 0.11 & 0.05 & 1 \\
\hline AY Cen & DCEP & 5.310 & 1.689 & 0.100 & 1864.4 & 303.0 & 1 & 2.821 & 0.15 & - & 1 \\
\hline AZ Cen & DCEPS & 3.212 & 2.137 & 0.158 & 2017.4 & 50.1 & 1 & 2.986 & 0.15 & - & 1 \\
\hline BB Cen & DCEPS & 3.998 & 3.610 & 0.363 & 3100.8 & 110.7 & 1 & 2.888 & 0.15 & - & 1 \\
\hline BB Gem & DCEP & 2.308 & 4.082 & 0.825 & 1135.9 & 49.5 & 1 & 3.123 & 0.16 & - & 1 \\
\hline BB Her & DCEP & 7.508 & 3.623 & 0.759 & 3122.0 & 153.2 & 8 & 2.684 & 0.05 & 0.32 & 1 \\
\hline BB Sgr & DCEP & 6.637 & 0.700 & 0.023 & 1529.1 & 30.8 & 1 & 2.800 & 0.15 & - & 1 \\
\hline BC Pup & DCEP & 3.544 & 6.500 & 1.109 & 938.2 & 64.4 & 2 & 2.760 & 0.11 & 0.13 & $4,(17)$ \\
\hline
\end{tabular}

Notes. Column 1: Name. Column 2: type (from Table 1 in G20). Nomenclature follows that used by the VSX (Watson et al. 2006) described in https://www.aavso.org/vsx/index.php?view=about.vartypes. Column 3: period (from G20). Column 4: distance (from G20). Column 5: error on distance (from G20). Column 6: luminosity (from G20). Column 7: error on Luminosity (from G20). The error is the fit error, and does not include the error on the distance. If the total error on $L$ is desired it can be calculated from $\sqrt{\sigma_{\mathrm{L}}^{2}+\Delta^{2}}$ with $\Delta=L \cdot\left(\left(1+\sigma_{\mathrm{d}} / d\right)^{2}-1\right)$. The

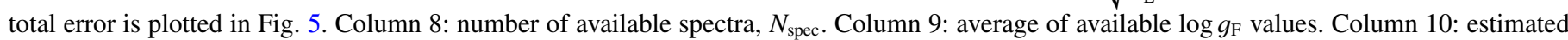
error on the average $\log g_{\mathrm{F}}$ value. This includes the error on $T_{\text {eff }}$ (when not given in the reference a conservative value of $100 \mathrm{~K}$ has been used) and the error on $\log g$ (assumed to be 0.15 dex, unless given specifically), divided by $\sqrt{N_{\text {spec }}}$. Column 11: difference between highest and lowest log $g_{\mathrm{F}}$ value. Column 12: references for $\log g$ and $T_{\text {eff }}$ values to calculate $\log g_{\mathrm{F}}$ and error. The full table is available at the CDS.

References. (1) Luck (2018), (2) Andrievsky et al. (1994), (3) Boyarchuk \& Lyubimkov (1981), (4) Luck et al. (2003), (5) Schmidt et al. (2011), (6) Andrievsky et al. (2002a), (7) Lemasle et al. (2008), (8) Andrievsky et al. (2013), (9) Kovtyukh et al. (2005), (10) Luck et al. (2006), (11) Yong et al. (2006), (12) Lemasle et al. (2015), (13) Romaniello et al. (2008), (14) Andrievsky et al. (2004), (15) Lemasle et al. (2007), (16) Andrievsky et al. (2002b), (17) Genovali et al. (2014), (18) Anders et al. (2019), (19) Martin et al. (2015), (20) Andrievsky et al. (2016), (21) Inno et al. (2019). Numbers in parentheses indicate references not considered. 